\title{
DISCRETENESS CRITERIA FOR MÖBIUS GROUPS ACTING ON $\overline{\mathbb{R}}^{n}$ II
}

\author{
LIU-LAN LI and XIAN-TAO WANG ${ }^{凶}$
}

(Received 23 June 2008)

\begin{abstract}
Jørgensen's famous inequality gives a necessary condition for a subgroup of $\operatorname{PSL}(2, \mathbb{C})$ to be discrete. It is also true that if Jørgensen's inequality holds for every nonelementary two-generator subgroup, the group is discrete. The sufficient condition has been generalized to many settings. In this paper, we continue the work of Wang, Li and Cao ('Discreteness criteria for Möbius groups acting on $\overline{\mathbb{R}}^{n}$, Israel J. Math. 150 (2005), 357-368) and find three more (infinite) discreteness criteria for groups acting on $\overline{\mathbb{R}}^{n}$; we also correct a linguistic ambiguity of their Theorem 3.3 where one of the necessary conditions might be vacuously fulfilled. The results of this paper are obtained by using known results regarding twogenerator subgroups and a careful analysis of the relation among the fixed point sets of various elements of the group.
\end{abstract}

2000 Mathematics subject classification: primary 30H40; secondary $20 \mathrm{H} 10$.

Keywords and phrases: discreteness criterion, two-generator Möbius group, parabolic element, loxodromic element, $g$-elliptic element.

\section{Introduction}

In this paper, we let $M\left(\overline{\mathbb{R}}^{n}\right)$ denote the full sense preserving Möbius group acting on $\overline{\mathbb{R}}^{n}$ and let $\overline{\mathbb{R}}=\mathbb{R} \cup\{\infty\}$, where $\mathbb{R}$ is the real field. We refer the reader to [4] for a fuller background and more detailed notation.

In [13], Jørgensen obtained a very useful necessary condition for two-generator Kleinian groups of $M\left(\overline{\mathbb{R}}^{2}\right)$, which is known as Jørgensen's inequality. As an application, he discussed the discreteness of subgroups of $M\left(\overline{\mathbb{R}}^{2}\right)$ or $M(\overline{\mathbb{R}})$ and obtained the following theorems (see [13, 14]).

THEOREM $J_{1}$. A nonelementary subgroup $G$ of $M\left(\overline{\mathbb{R}}^{2}\right)$ is discrete if and only if each two-generator subgroup of $G$ is discrete.

This research was partially supported by the Program for NCET (No. 04-0783), NSF of China (No. 10771059) and Hengyang Normal University (No. 08B06).

(C) 2009 Australian Mathematical Publishing Association Inc. 0004-9727/2009 \$16.00 
THEOREM $J_{2}$. A nonelementary subgroup $G$ of $M(\overline{\mathbb{R}})$ is discrete if and only if each cyclic subgroup of $G$ is discrete.

Furthermore, Gilman [10] proved the following theorem.

Theorem $G$. A nonelementary subgroup $G$ of $M(\overline{\mathbb{R}})$ is discrete if and only if every nonelementary subgroup generated by two hyperbolic elements of $G$ is discrete.

Wang and Yang [23] proved that Theorem $G$ also holds for subgroups in $M\left(\overline{\mathbb{R}}^{2}\right)$ and Tukia and Wang [18] proved the following theorem.

THEOREM $T W_{1}$. If a nonelementary subgroup $G$ of $M\left(\overline{\mathbb{R}}^{2}\right)$ contains an elliptic element of order at least three, then $G$ is discrete if and only if each nonelementary subgroup generated by two elliptic elements of $G$ is discrete.

Wang and Yang [24] proved the following theorem.

THEOREM WY. If a nonelementary subgroup $G$ of $M\left(\overline{\mathbb{R}}^{2}\right)$ contains a parabolic element, then $G$ is discrete if and only if each nonelementary subgroup generated by two parabolic elements of $G$ is discrete.

For any nontrivial element $f$ in $M\left(\overline{\mathbb{R}}^{n}\right)$, we let $\operatorname{Fix}(f)$ denote the set of its fixed point(s) in $\overline{\mathbb{R}}^{n}$. For any subgroup $G$ of $M\left(\overline{\mathbb{R}}^{n}\right)$, we let $H(G)$ denote the set of loxodromic elements of $G$ so that $H(G)=\{f \in G \mid f$ is loxodromic $\}$. Similarly let $P(G)$ denote the set of parabolic elements of $G$. We compare fixed points of arbitrary elements of $G$. Let $W Y(G)$ denote the set of the loxodromic fixing elements of $G$, that is, those elements of $G$ whose fixed point set contains the fixed points of every loxodromic element of $G$, and $W(G)$ the set of the parabolic fixing elements of $G$, that is, those elements of $G$ whose fixed point set contains the fixed point of each parabolic element of $G$. These subsets of $G$ are defined more precisely in Section 2. For now we note that these are not subgroups of $G$, but merely subsets.

DEFINITION 1.1. For any $f \in G$, let

$G_{f}=\{g \in G \mid g$ is conjugate to $f$ and the subgroup $\langle f, g\rangle$ is nonelementary $\} \cup\{f\}$,

where $\langle f, g\rangle$ denote the subgroup generated by $f$ and $g$.

As generalizations of Theorems $J_{1}, J_{2}, G, T W_{1}$ and $W Y$ to $M\left(\overline{\mathbb{R}}^{n}\right)$, one has the following results as obtained in [21].

TheOREM $W L C_{1}$. Let $G \subset \operatorname{PSL}\left(2, \Gamma_{n}\right)$ be nonelementary. Then $G$ is discrete if and only if $W Y(G)$ is discrete and each nonelementary subgroup generated by two elements of $G_{f}$ is discrete, where $f \in H(G)$.

THEOREM $W L C_{2}$. Let $G \subset \operatorname{PSL}\left(2, \Gamma_{n}\right)$ be nonelementary. If $G$ contains a parabolic element, then $G$ is discrete if and only if $W Y(G)$ is discrete and every nonelementary subgroup generated by two elements of $G_{f}$ is discrete, where $f \in P(G)$. 
We say that a subgroup $G \subset \operatorname{PSL}\left(2, \Gamma_{n}\right)$ satisfies the parabolic condition if $G$ contains no sequence $\left\{f_{i}\right\}$ such that each $f_{i}$ is parabolic and $f_{i} \rightarrow I$ as $i \rightarrow \infty$ (see [21]).

THEOREM $W L C_{3}$. Let $G \subset \operatorname{PSL}\left(2, \Gamma_{n}\right)$ be nonelementary and satisfy the parabolic condition. Suppose that $G$ contains a g-elliptic element $f$ such that $f^{2}$ is not an element of $W Y(G)$. Then $G$ is discrete if and only if $W Y(G)$ is discrete and each nonelementary subgroup of $G$ generated by two g-elliptic elements is discrete.

REMARK 1.2. We thank Shihai Yang for pointing out that in [21, Theorem 3.3] there is the possibility that the hypothesis on the discreteness of each nonelementary subgroup of $G$ generated by two $g$-elliptic elements be vacuously satisfied. Namely, there might not exist any nonelementary subgroups in $G$ generated by two $g$-elliptic elements. This was not assumed to be the case in the proof of the theorem. Here Theorem $W L C_{3}$ is a corrected version of [21, Theorem 3.3].

We the reader refer to $[1,6,7,9,15,29]$ for further discussions on this line of work. In [18] Tukia and Wang proved the following theorems.

THEOREM $T W_{2}$. Let $G$ be a nonelementary subgroup of $M\left(\overline{\mathbb{R}}^{2}\right)$. If $G$ contains an elliptic element of order at least three, then $G$ is discrete if and only if each nonelementary subgroup of $G$ generated by an elliptic element and a loxodromic element is discrete.

THEOREM $T W_{3}$. Let $G$ be a nonelementary subgroup of $M\left(\overline{\mathbb{R}}^{2}\right)$ containing parabolic elements. Then $G$ is discrete if and only if each nonelementary subgroup of $G$ generated by a parabolic element and a loxodromic element is discrete.

In [30] Yang proved the following result which provides an affirmative answer to the open problem raised in [18].

THEOREM $Y$. Let $G$ be a nonelementary subgroup of $\operatorname{SL}(2, \mathbb{C})$ containing parabolic and elliptic elements. Then $G$ is discrete if and only if each subgroup of $G$ generated by a parabolic element and an elliptic element is discrete.

We refer the reader to $[3,5,8,12]$ for related investigations in this direction.

The main aim of this paper is to generalize Theorems $T W_{2}, T W_{3}$ and $Y$ to the $n$-dimensional case. Our main results are Theorems 3.1, 3.2 and 3.3. They are stated in Section 3 and proved in Section 6.

\section{Preliminaries}

We need the following preliminaries, see $[2,22,27]$ for more details.

Let $\Gamma_{n}$ denote the $n$-dimensional Clifford group, $\operatorname{SL}\left(2, \Gamma_{n}\right)$ the group of all $n$-dimensional Clifford matrices and

$$
\operatorname{PSL}\left(2, \Gamma_{n}\right)=\operatorname{SL}\left(2, \Gamma_{n}\right) /\{ \pm I\},
$$

where $I$ is the unit matrix. 
Let $A$ be defined by

$$
A=\left(\begin{array}{ll}
a & b \\
c & d
\end{array}\right) \in \operatorname{PSL}\left(2, \Gamma_{n}\right)
$$

corresponding to the mapping in $\overline{\mathbb{R}}^{n}$ :

$$
x \mapsto A x=(a x+b)(c x+d)^{-1} .
$$

It is known that this is an isomorphism between $\operatorname{PSL}\left(2, \Gamma_{n}\right)$ and $M\left(\overline{\mathbb{R}}^{n}\right)$ (see [2]). In the following, we identify the element in $M\left(\overline{\mathbb{R}}^{n}\right)$ and its corresponding element in $\operatorname{PSL}\left(2, \Gamma_{n}\right)$.

For $f \in \operatorname{PSL}\left(2, \Gamma_{n}\right)$, let $\tilde{f}$ denote the Poincaré extension of $f$ to $\mathbb{H}^{n+1}$ (see [4]),

$$
\operatorname{Fix}(\tilde{f})=\left\{z \in \mathbb{H}^{n+1} \mid \tilde{f}(z)=z\right\}
$$

and let $\operatorname{Card}(M)$ denote the cardinality of the set $M$.

Now, we classify the elements of $\operatorname{PSL}\left(2, \Gamma_{n}\right)$ as follows. A nontrivial element $f \in \operatorname{PSL}\left(2, \Gamma_{n}\right)$ is called:

(1) fixed point free if $\operatorname{Card}[\operatorname{Fix}(f)]=0$;

(2) loxodromic if $\operatorname{Card}[\operatorname{Fix}(f)]>0$ and $f$ can be conjugate in $\operatorname{PSL}\left(2, \Gamma_{n}\right)$ to $\left(\begin{array}{cc}r \lambda & 0 \\ 0 & r^{-1} \lambda^{\prime}\end{array}\right)$, where $r>0, r \neq 1, \lambda \in \Gamma_{n}$ and $|\lambda|=1$;

(3) parabolic if $\operatorname{Card}[\operatorname{Fix}(f)]>0$ and $f$ can be conjugate in $\operatorname{PSL}\left(2, \Gamma_{n}\right)$ to $\left(\begin{array}{ll}a & b \\ 0 & a^{\prime}\end{array}\right)$, where $a, b \in \Gamma_{n},|a|=1, b \neq 0$ and $a b=b a^{\prime}$;

(4) elliptic if $\operatorname{Card}[\operatorname{Fix}(f)]>0$ and $f$ can be conjugate in $\operatorname{PSL}\left(2, \Gamma_{n}\right)$ to $\left(\begin{array}{ll}u & 0 \\ 0 & u^{\prime}\end{array}\right)$, $u \in \Gamma_{n},|u|=1$ and $u \notin \mathbb{R}$.

We call $f$ g-elliptic if it is elliptic or fixed point free.

REMARK 2.1. When $n=1$ or $n=2$, since $M\left(\overline{\mathbb{R}}^{n}\right)$ contains no fixed-point-free element, we see that each $g$-elliptic element in $M\left(\overline{\mathbb{R}}^{n}\right)$ is elliptic.

PROPOSITION 2.2. For a nontrivial element $f \in \operatorname{PSL}\left(2, \Gamma_{n}\right)$ :

(i) $\quad f$ is fixed point free if and only if $\operatorname{Card}[\operatorname{Fix}(\tilde{f})]=1 ; f$ is elliptic if and only if $\operatorname{Card}[\operatorname{Fix}(\tilde{f})]>1$;

(ii) $\operatorname{PSL}\left(2, \Gamma_{n}\right)$ contains a fixed-point-free element if and only if $n$ is odd and $n \geq 3$.

It follows from Proposition 2.2 that for any nontrivial element $f$, it is $g$-elliptic if and only if $\operatorname{Fix}(\widetilde{f}) \neq \emptyset$.

A subgroup $G$ of $\operatorname{PSL}\left(2, \Gamma_{n}\right)$ is called elementary if it has a finite $G$-orbit in $\overline{\mathbb{H}}^{n+1}=\mathbb{H}^{n+1} \cup \overline{\mathbb{R}}^{n}$ (see [4]). Otherwise, we call $G$ a nonelementary subgroup of $\operatorname{PSL}\left(2, \Gamma_{n}\right)$.

The limit set of $G$ is

$$
L(G)=\overline{\mathbb{R}}^{n} \cap \operatorname{cl}\left(G_{z}\right)
$$

where $z \in \mathbb{H}^{n+1}, G_{z}=\{\tilde{g}(z) \mid g \in G\}$ and cl denotes the closure. Then $L(G)$ is independent of the choice of $z$ (see [17]). 
A subgroup $G$ of PSL $\left(2, \Gamma_{n}\right)$ is called Kleinian if it is nonelementary and discrete.

From the discussions in [28], Remark $B_{1}$ and the proof of Lemma $B_{2}$ in [17], we easily obtain the following.

LEMMA 2.3. We have the following results.

(i) If $G$ contains a loxodromic element, then $G$ is elementary if and only if it fixes a point in $\overline{\mathbb{R}}^{n}$ or preserves a set consisting of two points in $\overline{\mathbb{R}}^{n}$.

(ii) If $G$ contains a parabolic element but no loxodromic element, then $G$ is elementary if and only if it fixes a point in $\overline{\mathbb{R}}^{n}$.

(iii) If $G$ is purely g-elliptic, that is, each nontrivial element of $G$ is g-elliptic, then G fixes a point in $\overline{\mathbb{H}}^{n+1}$.

REMARK 2.4. The case that $G$ fixes only one point in $\overline{\mathbb{R}}^{n}$ in Lemma 2.3(iii) can occur when $n \geq 4$ (see [28]).

In view of Lemma 2.3 and Remark 2.4, we obtain the following corollary.

COROLlary 2.5. If the elements of $G \subset \operatorname{PSL}\left(2, \Gamma_{n}\right)$ have no common fixed points in $\overline{\mathbb{R}}^{n}$, then $G$ is purely g-elliptic if and only if the elements of $G$ have a common fixed point in $\mathbb{H}^{n+1}$.

For

$$
g_{r}=\left(\begin{array}{ll}
a_{r} & b_{r} \\
c_{r} & d_{r}
\end{array}\right) \in \operatorname{PSL}\left(2, \Gamma_{n}\right) \quad(r=1,2),
$$

we define

$$
\left\|g_{1}-g_{2}\right\|=\left(\left|a_{1}-a_{2}\right|^{2}+\left|b_{1}-b_{2}\right|^{2}+\left|c_{1}-c_{2}\right|^{2}+\left|d_{1}-d_{2}\right|^{2}\right)^{\frac{1}{2}} .
$$

The following lemma is crucial for our investigation.

LEMMA 2.6 (Waterman [27]). Let $f, g \in \operatorname{PSL}\left(2, \Gamma_{n}\right)$. If $\langle f, g\rangle$ is a Kleinian group, then

$$
\|f-I\| \cdot\|g-I\| \geq \frac{1}{32} .
$$

For a nonelementary subgroup $G \subset \operatorname{PSL}\left(2, \Gamma_{n}\right)$, let

$$
W Y(G)=\{h \in G \mid \operatorname{Fix}(f) \subset \operatorname{Fix}(h) \text { for all } f \in H(G)\} \quad \text { (see [22]) }
$$

and

$$
W(G)=\{h \in G \mid \operatorname{Fix}(f) \subset \operatorname{Fix}(h) \text { for all } f \in P(G)\} .
$$

PROPOSITION 2.7. If $G$ is nonelementary, then:

(i) $H(G) \neq \emptyset($ see $[25,26])$;

(ii) $W(G)=W Y(G)$ provided that $G$ contains some parabolic element;

(iii) $W Y(G)=L(I)$ (see [6] for the definition);

(iv) $W Y(G)$ is discrete if and only if $W Y(G)$ is finite. 
REMARK 2.8. When $n=1$ or $2, W Y(G)=L(I)=\{I\}(=W(G)$ if $P(G) \neq \emptyset)$ provided that $G \subset \operatorname{PSL}\left(2, \Gamma_{n}\right)$ is nonelementary, see [22].

Let $G \subset \operatorname{PSL}\left(2, \Gamma_{n}\right)$ be nonelementary, and $\sigma(L(G))$ be the sphere of the smallest dimension containing the limit set $L(G)$. Then the dimension of $\sigma(L(G))$ is at least one. By conjugation, we may assume that $\sigma(L(G))=\overline{\mathbb{R}}^{k}$, where $1 \leq k \leq n$. Then we have the following decomposition theorem.

THEOREM 2.9. Let $G \subset \operatorname{PSL}\left(2, \Gamma_{n}\right)$ be nonelementary with $\sigma(L(G))=\overline{\mathbb{R}}^{k} \quad(1 \leq$ $k \leq n)$. Then for every element $g \in G, g=\widetilde{g}_{1} \circ g_{0}=g_{0} \circ \widetilde{g}_{1}$, where $g_{1}=\left.g\right|_{\mathbb{R}^{k}}$, the restriction of $g$ to $\overline{\mathbb{R}}^{k}, \widetilde{g_{1}}$ denotes the Poincaré extension of $g_{1}$ to $\overline{\mathbb{R}}^{n}$ and $g_{0}$ is a rotation with $\left.g_{0}\right|_{\overline{\mathbb{R}}^{k}}=I$.

PRoOF. If $g(\infty)=\infty$, then $g(x)=t A x+b$, where $t>0, A \in O(n), \operatorname{det}(A)=1$ and $b \in \mathbb{R}^{n}$. Since $g\left(\mathbb{R}^{k} \times\{0\}\right)=\mathbb{R}^{k} \times\{0\}$, we know that $b=\left(b_{1}, b_{2}, \ldots, b_{k}, 0, \ldots, 0\right)$ $\in \mathbb{R}^{k} \times\{0\}$ and

$$
A=\left(\begin{array}{cc}
A_{k} & 0 \\
0 & A_{n-k}
\end{array}\right)
$$

where $\{0\}=(\underbrace{0, \ldots, 0}_{n-k})$,

$$
\mathbb{R}^{k} \times\{0\}=\left\{x \in \mathbb{R}^{n} \mid x=\left(x_{1}, \ldots, x_{k}, 0, \ldots, 0\right), x_{i} \in \mathbb{R}, i=1, \ldots, k\right\},
$$

$A_{k} \in O(k)$ and $A_{n-k} \in O(n-k)$. Let $g_{1}(y)=t A_{k} y+b^{\prime}$ and

$$
g_{0}=\left(\begin{array}{cc}
E_{k} & 0 \\
0 & A_{n-k}
\end{array}\right) \text {, }
$$

where $y \in \overline{\mathbb{R}}^{k}, b^{\prime}=\left.b\right|_{\overline{\mathbb{R}}^{k}}=\left(b_{1}, b_{2}, \ldots, b_{k}\right)$ and $E_{k}$ is the $k \times k$ identity matrix. The conclusion follows for this case.

If $g(\infty) \neq \infty$, then $g(x)=t A\left((x-b) /|x-b|^{2}\right)+a$ (see [4]). Since $g$ preserves the subspace $\mathbb{R}^{k} \times\{0\}$, we have that $a, b \in \mathbb{R}^{k} \times\{0\}$ and

$$
A=\left(\begin{array}{cc}
A_{k} & 0 \\
0 & A_{n-k}
\end{array}\right),
$$

where $A_{k} \in O(k)$ and $A_{n-k} \in O(n-k)$. Let $g_{1}(y)=t A_{k}\left(\left(y-b^{\prime}\right) /\left|y-b^{\prime}\right|^{2}\right)+a^{\prime}$ and

$$
g_{0}=\left(\begin{array}{cc}
E_{k} & 0 \\
0 & A_{n-k}
\end{array}\right),
$$

where $y \in \overline{\mathbb{R}}^{k}$ and $a^{\prime}=\left.a\right|_{\overline{\mathbb{R}}^{k}}$. It follows that $g=\widetilde{g_{1}} \circ g_{0}=g_{0} \circ \widetilde{g_{1}}$.

For a nonelementary subgroup $G \subset \operatorname{PSL}\left(2, \Gamma_{n}\right)$, as in [19], we define a homomorphism $\Phi$ from $G$ onto $\Phi(G)$ given by $\Phi(g)=\left.g\right|_{\sigma(L(G))}=g_{1}$ for every $g \in G$, that is, $\Phi(g)$ is the restriction of $g$ to $\sigma(L(G))$. Then we easily obtain the following. 
PROPOSITION 2.10. We have the following results:

(i) $W Y(G)=\{g \in G \mid \Phi(g)=I\}$, which is a subgroup of $G$;

(ii) when $P(G) \neq \emptyset, W(G)=\{g \in G \mid \Phi(g)=I\}$, which is also a subgroup of $G$.

Since $\sigma(L(G))=\overline{\mathbb{R}}^{k}$, the following are obvious.

LEMMA 2.11. An element $f$ in $G$ is loxodromic (respectively parabolic) if and only if $\Phi^{2}(f) \in \operatorname{PSL}\left(2, \Gamma_{k}\right)$ is loxodromic (respectively parabolic).

LEMMA 2.12. We have the following results.

(i) If $f \in G$ is $g$-elliptic, then $\Phi^{2}(f) \in \operatorname{PSL}\left(2, \Gamma_{k}\right)$ is g-elliptic or $I$.

(ii) If $\Phi^{2}(f) \in \operatorname{PSL}\left(2, \Gamma_{k}\right)$ is $g$-elliptic, then the corresponding element $f$ of $\Phi(f)$ in $G$ must be g-elliptic.

We now recall a result from [19], which is a generalization of [16, Proposition p. 246] to $M\left(\overline{\mathbb{R}}^{n}\right)$.

LEMMA 2.13. Let $G \subset \operatorname{PSL}\left(2, \Gamma_{n}\right)$ be nonelementary. Suppose that $\sigma(L(G))=$ $\overline{\mathbb{R}}^{k}(1 \leq k \leq n)$. Then:

(i) $G$ is discrete;

(ii) $\operatorname{Ker} \Phi$ is not discrete but $\Phi(G)$ is discrete; or

(iii) $\Phi(G) \cap \operatorname{PSL}\left(2, \Gamma_{k}\right)$ is a dense subgroup of $\operatorname{PSL}\left(2, \Gamma_{k}\right)$.

Moreover, we prove the following result for subgroups in $M\left(\overline{\mathbb{R}}^{2}\right)$.

Proposition 2.14. Let $G \subset \operatorname{PSL}(2, \mathbb{C})$ be nonelementary and nondiscrete. If $G$ contains an elliptic element of order at least three, then $G$ contains a nonelementary and nondiscrete subgroup $\left\langle g_{1}, g_{2}\right\rangle$ generated by $g_{1}$ and $g_{2}$, where $g_{1}$ is elliptic and $g_{2}$ is loxodromic.

The following easy fact plays an important role in the proof of Proposition 2.14.

LEMMA 2.15. Let $g$ be an elliptic element of order at least three in $\operatorname{PSL}(2, \mathbb{C})$, and let $\left\{f_{i}\right\} \subset \operatorname{PSL}(2, \mathbb{C})$ be a sequence of distinct elements such that

$$
f_{i} \rightarrow I \quad \text { as } i \rightarrow \infty
$$

If $\left\langle g, f_{i}\right\rangle$ is nonelementary for each $i$, then $\left[g, f_{i}\right] \neq I$,

$$
\left[g, f_{i}\right] \rightarrow I
$$

and $\left[g, f_{i}\right]$ is not parabolic for all sufficiently large $i$.

Proof of Proposition 2.14. Since $G$ is not discrete, we see that there is a sequence $\left\{f_{i}\right\}$ of distinct elements of $G$ converging to the identity. By passing to a subsequence (denoted in the same manner), we may assume that each $f_{i}$ is elliptic, or each $f_{i}$ is loxodromic, or each $f_{i}$ is parabolic. 
If each $f_{i}$ is elliptic, then, by choosing a subsequence if needed (still denoted in the same way), we know that there exists a loxodromic element $h$ in $G$ such that

$$
\operatorname{Fix}(h) \cap \operatorname{Fix}\left(f_{i}\right)=\emptyset
$$

for all sufficiently large $i$. Then $\left\langle h, f_{i}\right\rangle$ is nonelementary but not discrete by Jørgensen's inequality.

If each $f_{i}$ is loxodromic, then, by choosing a subsequence if needed (still denoted in the same manner), we know that there exists an elliptic element $h$ of order at least three such that

$$
\operatorname{Fix}(h) \cap \operatorname{Fix}\left(f_{i}\right)=\varnothing
$$

for all sufficiently large $i$. By Jørgensen's inequality, we know that $\left\langle h, f_{i}\right\rangle$ is nonelementary and nondiscrete.

If each $f_{i}$ is parabolic, by passing to a suitable subsequence (denoted in the same manner), we may assume that $\operatorname{Fix}\left(f_{i}\right)$ tends in the Hausdorff metric to a one-point subset of $\overline{\mathbb{C}}$. Then we can find an elliptic element $g$ of order at least three such that

$$
\operatorname{Fix}(g) \cap \operatorname{Fix}\left(f_{i}\right)=\emptyset
$$

for large enough $i$.

This implies the nonelementariness of $\left\langle g, f_{i}\right\rangle$. It follows from Lemma 2.15 that $\left[g, f_{i}\right] \neq I,\left[g, f_{i}\right]$ is not parabolic for all sufficiently large $i$, and

$$
\left[g, f_{i}\right] \rightarrow I \quad \text { as } i \rightarrow \infty
$$

By choosing a subsequence if needed, we may assume that each $\left[g, f_{i}\right]$ is elliptic or each $\left[g, f_{i}\right]$ is loxodromic. By the above discussions, we know that the conclusion holds.

From Proposition 2.14 the following results follow easily.

Corollary 2.16. Let $G \subset \operatorname{PSL}(2, \mathbb{C})$ be nonelementary and contain an elliptic element with the order at least three. Then $G$ is discrete if and only if each nonelementary subgroup of $G$ generated by a loxodromic element and an elliptic element is discrete.

Corollary 2.17. Let $G \subset \operatorname{PSL}(2, \mathbb{C})$ be nonelementary and contain an elliptic element with order at least three. If $G$ is discrete or each nonelementary subgroup generated by a loxodromic element and an elliptic element of $G$ is discrete, then $G$ satisfies the parabolic condition.

REMARK 2.18. Corollary 2.16 provides an alternate proof of Theorem $T W_{2}$.

\section{Discreteness criteria for subgroups of $\operatorname{PSL}\left(2, \Gamma_{n}\right)$}

The following are our main results which are proved in Section 6. 
Theorem 3.1. Let $G \subset \operatorname{PSL}\left(2, \Gamma_{n}\right)$ be nonelementary and satisfy the parabolic condition. Suppose that $G$ contains a g-elliptic element $f$ such that $f^{2}$ is not an element of $W Y(G)$. Then $G$ is discrete if and only if:

(i) $W Y(G)$ is discrete; and

(ii) each nonelementary subgroup of $G$ generated by a loxodromic element and a g-elliptic element is discrete.

Theorem 3.2. Let $G \subset \operatorname{PSL}\left(2, \Gamma_{n}\right)$ be nonelementary. Suppose that $G$ contains a parabolic element. Then $G$ is discrete if and only if:

(i) $W Y(G)(=W(G))$ is discrete; and

(ii) every nonelementary subgroup of $G$ generated by a loxodromic element and a parabolic element is discrete.

THeOREM 3.3. Let $G \subset \operatorname{PSL}\left(2, \Gamma_{n}\right)$ be nonelementary. Suppose that $G$ contains a parabolic element and a g-elliptic element which is not an element of $W Y(G)(=$ $W(G))$. Then $G$ is discrete if and only if:

(i) $W Y(G)$ is discrete; and

(ii) each nonelementary subgroup of $G$ generated by a g-elliptic element and a parabolic element is discrete.

REMARK 3.4. The examples in $[19,22]$ show that the condition ' $W Y(G)$ being discrete' in the above theorems cannot be removed.

REMARK 3.5. When $n=1$ or 2, by Remark 2.8 and Corollary 2.17, Theorem 3.1 coincides with Theorem $T W_{2}$. When $n \geq 3$, Theorem 3.1 is a generalization of Theorem $T W_{2}$.

Remark 3.6. When $n=2$, by Remark 2.8, Theorem 3.2 coincides with Theorem $T W_{3}$. When $n \geq 3$, Theorem 3.2 is a generalization of Theorem $T W_{3}$. Theorem 3.3 is a generalization of Theorem $Y$.

\section{Existence of three classes of two-generator subgroups}

The main aim of this section is to prove the following three lemmas.

Lemma 4.1. Let $G \subset \operatorname{PSL}\left(2, \Gamma_{n}\right)$ be nonelementary, which contains a g-elliptic element $f$ such that $f^{2}$ is not an element of $W Y(G)$. Then there are at least two loxodromic elements $h_{r}(r=1,2)$ in $G$ such that

$$
\operatorname{Fix}\left(h_{1}\right) \cap \operatorname{Fix}\left(h_{2}\right)=\emptyset \quad \text { and } \operatorname{Fix}\left(h_{r}\right) \cap \operatorname{Fix}\left(f^{2}\right)=\emptyset .
$$

That is, the subgroups $\left\langle f, h_{r}\right\rangle$ are nonelementary.

Lemma 4.2. Let $G \subset \operatorname{PSL}\left(2, \Gamma_{n}\right)$ be nonelementary. If $G$ contains a g-elliptic element $f$ such that $f^{2}$ is not an element of $W Y(G)$, then $G$ contains a loxodromic element $g$ such that the subgroup $\left\langle f, g f g^{-1}\right\rangle$ is nonelementary. 
The following is obvious.

LEMMA 4.3. Let $G \subset \operatorname{PSL}\left(2, \Gamma_{n}\right)$ be nonelementary. Suppose that $G$ contains a parabolic element and a g-elliptic element which is not an element of $W Y(G)$. Then $G$ contains a nonelementary subgroup generated by a g-elliptic element and a parabolic element.

REMARK 4.4. Lemma 4.1 shows that under the hypotheses of Theorem 3.1, $G$ contains a nonelementary subgroup which is generated by a loxodromic element and a $g$-elliptic element.

REMARK 4.5. Lemma 4.2 shows that under the hypotheses of Theorem $W L C_{3}, G$ contains a nonelementary subgroup which is generated by two $g$-elliptic elements.

REMARK 4.6. Lemma 4.3 shows that under the hypotheses of Theorem 3.3, $G$ contains a nonelementary subgroup which is generated by a $g$-elliptic element and a parabolic element.

The following result is crucial for our following discussions.

LEMMA 4.7. Let $G \subset \operatorname{PSL}\left(2, \Gamma_{n}\right)$ be nonelementary. If $G$ contains a g-elliptic element $f$ which is not an element of $W Y(G)$, then $G$ contains a loxodromic element $h$ such that $\operatorname{Fix}(h) \cap \operatorname{Fix}(f)=\emptyset$.

Proof. Since $f \notin W Y(G)$, there must be a loxodromic element $h \in G$ such that the attractive fixed point of $h$ is not fixed by $f$.

If the repulsive fixed point of $h$ is also not fixed by $f$, then $\operatorname{Fix}(h) \cap \operatorname{Fix}(f)=\emptyset$.

If the repulsive fixed point of $h$ is fixed by $f$, then there is another loxodromic element $g$ in $G$ such that $\operatorname{Fix}(h) \cap \operatorname{Fix}(g)=\emptyset$ since $G$ is nonelementary.

Let

$$
h_{s}=h^{s} \circ g \circ h^{-s} \text {. }
$$

Then for sufficiently large $s, \operatorname{Fix}\left(h_{s}\right) \cap \operatorname{Fix}(f)=\emptyset$.

By replacing $h$ with $h_{s}$, we have proved the conclusion.

Proof of LEMma 4.1. Lemma 4.7 implies that there exists a loxodromic element $h$ in $G$ such that $\operatorname{Fix}(h) \cap \operatorname{Fix}\left(f^{2}\right)=\emptyset$. It follows from the nonelementariness of $G$ that there exists a loxodromic element $g$ in $G$ with $\operatorname{Fix}(g) \cap \operatorname{Fix}(h)=\emptyset$. Then there is a natural number $K$ satisfying that for any $k>K, \operatorname{Fix}\left(h^{k} g h^{-k}\right) \cap \operatorname{Fix}\left(f^{2}\right)=\emptyset$. Obviously, $\left\langle h^{k} g h^{-k}, f\right\rangle$ is nonelementry. The result follows.

The proof of Lemma 4.2 needs the following result.

LEMMA 4.8. Let $G \subset \operatorname{PSL}\left(2, \Gamma_{n}\right)$ be nonelementary. If $G$ contains a g-elliptic element $f$ which is not an element of $W Y(G)$, then $G$ contains a loxodromic element $g$ such that $\operatorname{Fix}\left(g f g^{-1}\right) \cap \operatorname{Fix}(f)=\emptyset$ and $\operatorname{Fix}\left(\widetilde{g f g^{-1}}\right) \cap \operatorname{Fix}(\tilde{f})=\emptyset . \quad($ Recall that $\tilde{f}$ denotes the Poincaré extension of $f$ in $\mathbb{H}^{n+1}$.) 
PROOF. Since $f \notin W Y(G)$, by Lemma 4.7, there must be a loxodromic element $h \in G$ such that $\operatorname{Fix}(h) \cap \operatorname{Fix}(f)=\emptyset$.

Let $f_{k}=h^{k} \circ f \circ h^{-k}$. Then for sufficiently large $k$,

$$
\operatorname{Fix}\left(f_{k}\right) \cap \operatorname{Fix}(f)=\emptyset \quad \text { and } \quad \operatorname{Fix}\left(\tilde{f}_{k}\right) \cap \operatorname{Fix}(\tilde{f})=\emptyset .
$$

By letting $g=h^{k}$ for some large enough $k$, we see that the lemma holds.

Proof of Lemma 4.2. Since $f^{2} \notin W Y(G)$, by Lemma 4.8 , there must be a loxodromic element $g \in G$ such that

$$
\operatorname{Fix}\left(g f^{2} g^{-1}\right) \cap \operatorname{Fix}\left(f^{2}\right)=\emptyset \quad \text { and } \quad \operatorname{Fix}\left(\widetilde{g f^{2} g^{-1}}\right) \cap \operatorname{Fix}\left(\tilde{f}^{2}\right)=\emptyset
$$

These imply that

$$
\operatorname{Fix}\left(g f g^{-1}\right) \cap \operatorname{Fix}(f)=\emptyset \quad \text { and } \quad \operatorname{Fix}\left(\widetilde{g f g^{-1}}\right) \cap \operatorname{Fix}(\tilde{f})=\emptyset .
$$

Hence, the subgroup $\left\langle f, g f g^{-1}\right\rangle$ is nonelementary.

\section{Several Propositions}

The main results of this section are the following which are useful for the proofs in the next section.

Proposition 5.1. Let $G \subset \operatorname{PSL}\left(2, \Gamma_{n}\right)$ be nonelementary. Suppose that $W Y(G)$ is discrete and each nonelementary subgroup of $G$ generated by a loxodromic element and a g-elliptic element is discrete. Then $G$ contains no sequence $\left\{f_{i}\right\}$ such that each $f_{i}$ is g-elliptic and

$$
f_{i} \rightarrow I \quad \text { as } i \rightarrow \infty \text {. }
$$

Proposition 5.2. Let $G \subset \operatorname{PSL}\left(2, \Gamma_{n}\right)$ be nonelementary, which contains a parabolic element. Suppose that $W Y(G)$ is discrete and every nonelementary subgroup of $G$ generated by a loxodromic element and a parabolic element is discrete. Then $G$ contains no sequence $\left\{f_{i}\right\}$ such that each $f_{i}$ is not $g$-elliptic and

$$
f_{i} \rightarrow I \quad \text { as } i \rightarrow \infty
$$

Proposition 5.3. Let $G \subset \operatorname{PSL}\left(2, \Gamma_{n}\right)$ be nonelementary. Suppose that $W Y(G)$ is discrete and each nonelementary subgroup of $G$ generated by a $g$-elliptic element and a parabolic element is discrete. Then $G$ contains no sequence $\left\{f_{i}\right\}$ such that each $f_{i}$ is $g$-elliptic and

$$
f_{i} \rightarrow I \quad \text { as } i \rightarrow \infty
$$


Proof of Proposition 5.1. Suppose, in contrast, that $G$ contains such a sequence. Without loss of generality, we may assume that $\sigma(L(G))=\overline{\mathbb{R}}^{k}$, where $1 \leq k \leq n$. Choose $x_{j} \in L(G)$ and accordingly open balls $U_{j}$ in $\overline{\mathbb{R}}^{n}(j=1,2, \ldots, k+2)$ such that $x_{j} \in U_{j}, U_{j} \cap U_{s}=\emptyset$ whenever $j \neq s$ and for any $a_{j} \in U_{j}$, there exists only one $k$-sphere $S\left(a_{1}, \ldots, a_{k+2}\right)$ containing $a_{1}, \ldots, a_{k+2}$.

Since $W Y(G)$ is finite, there is a ball $U_{j_{0}}$ such that $\operatorname{Fix}\left(f_{i}^{2}\right) \cap U_{j_{0}}=\emptyset$ for large $i$, where $j_{0} \in\{1,2, \ldots, k+2\}$. Since $U_{j_{0}} \cap L(G) \neq \emptyset$, there is a loxodromic element $g$ with $\operatorname{Fix}(g) \subset U_{j_{0}}$. Thus, $\operatorname{Fix}(g) \cap \operatorname{Fix}\left(f_{i}^{2}\right)=\emptyset$ for all large $i$. It follows that $\left\langle g, f_{i}\right\rangle$ is nonelementary and hence discrete. This violates Lemma 2.6 since $f_{i} \rightarrow I$ as $i \rightarrow \infty$.

Proof of Proposition 5.2. Suppose, in contrast, that $G$ contains such a sequence. By choosing a subsequence if necessary, we may assume that each $f_{i}$ is parabolic (respectively loxodromic). Then there is a loxodromic (respectively parabolic) element $g$ so that $\operatorname{Fix}(g) \cap \operatorname{Fix}\left(f_{i}\right)=\emptyset$ for large enough $i$ by passing to a suitable subsequence of $\left\{f_{i}\right\}$ (still denoted in the same manner). Therefore, $\left\langle g, f_{i}\right\rangle$ is nonelementary and hence discrete for large $i$. By Lemma 2.6, this is a contradiction since $f_{i} \rightarrow I$ as $i \rightarrow \infty$.

Proof of Proposition 5.3. Suppose, in contrast, that $G$ contains such a sequence. Since $W(G)$ is finite, there is a parabolic element $g$ so that $\operatorname{Fix}(g) \cap \operatorname{Fix}\left(f_{i}\right)=\emptyset$ for all large enough $i$ by passing to a suitable subsequence if needed, which is denoted in the same way. Since

$$
\|g-I\| \cdot\left\|f_{i}-I\right\|<\frac{1}{32}
$$

for large $i$, Lemma 2.6 implies that $\left\langle g, f_{i}\right\rangle$ is elementary. This yields that $\operatorname{Fix}(g) \cap$ $\operatorname{Fix}\left(f_{i}\right) \neq \emptyset$. This is the desired contradiction.

\section{The proofs of the main results}

First, let us introduce two lemmas which are needed in the proof of Theorem 3.3.

LEMMA 6.1 (Wang [20]). Let $\left\{f_{i}\right\}$ and $\left\{g_{i}\right\}$ be two sequences of $M\left(\overline{\mathbb{R}}^{n}\right)$, which converge to $f$ and $g$, respectively. Suppose that each group $\left\langle f_{i}, g_{i}\right\rangle$ is a Kleinian group and each $f_{i}$ is of infinite order. Then $f$ is of infinite order and $\langle f, g\rangle$ is a Kleinian group if $\left\{\left\langle f_{i}, g_{i}\right\rangle\right\}$ satisfies the E-condition.

Here, we say that a sequence $\left\{G_{i}\right\}$ of subgroups of $M\left(\overline{\mathbb{R}}^{n}\right)$ satisfies the $E$-condition if any sequence $\left\{f_{i_{k}}\right\}\left(f_{i_{k}} \in G_{i_{k}}\left(\in\left\{G_{i}\right\}\right)\right)$ satisfying that for each $k$, $\operatorname{Card}\left[\operatorname{Fix}\left(f_{i_{k}}\right)\right]=$ $\infty$ and $f_{i_{k}} \rightarrow I$ as $k \rightarrow \infty$ has uniformly bounded torsion, that is, there exists a positive number $M$ such that for each $k$, the order $\operatorname{ord}\left(f_{i_{k}}\right)$ of $f_{i_{k}}$ satisfies that $\operatorname{ord}\left(f_{i_{k}}\right) \leq M$ or $\operatorname{ord}\left(f_{i_{k}}\right)=\infty$.

LEMMA 6.2 (Hersonsky [11]). Let $M=\left(\begin{array}{ll}a & b \\ c & d\end{array}\right)$ be an element of $\operatorname{SL}\left(2, \Gamma_{n}\right)$, where $c \neq 0$, and let $U=\left(\begin{array}{ll}1 & 1 \\ 0 & 1\end{array}\right)$. If the group $\langle M, U\rangle$ is discrete, then $|c| \geq 1$. 
Proof of Theorem 3.1. Suppose that $G$ is not discrete. Then Proposition 5.1 and the hypothesis $G$ satisfying the parabolic condition show that $G$ contains a sequence $\left\{f_{i}\right\}$ such that each $f_{i}$ is loxodromic and

$$
f_{i} \rightarrow I \quad \text { as } i \rightarrow \infty
$$

Then, by choosing a subsequence if necessary, we may assume that $\operatorname{Fix}\left(f_{i}\right)$ tends in the Hausdorff metric toward a one- or two-point set $Y \subset \overline{\mathbb{R}}^{n}$.

We now divide our proof into the following cases.

CASE I: $Y \subset \operatorname{Fix}\left(f^{2}\right)$. By Lemma 4.7, there is a loxodromic element $g \in G$ such that

$$
\operatorname{Fix}(g) \cap \operatorname{Fix}\left(f^{2}\right)=\emptyset .
$$

For large enough $k$, we let $h=g^{k} f^{2} g^{-k}$. Then the subgroup $\left\langle h, f_{i}\right\rangle$ is nonelementary for each large $i$.

CASE II: $Y=\{x, y\}, f^{2}(x)=x$ and $f^{2}(y) \neq y$. By Lemma 4.1, there are two loxodromic elements $h_{r}(r=1,2)$ in $G$ such that

$$
\operatorname{Fix}\left(h_{1}\right) \cap \operatorname{Fix}\left(h_{2}\right)=\emptyset \quad \text { and } \quad \operatorname{Fix}\left(h_{r}\right) \cap \operatorname{Fix}\left(f^{2}\right)=\emptyset .
$$

If $h_{1}(y)=y$, then $h_{2}(y) \neq y$, that is, $\operatorname{Fix}\left(h_{2}\right) \cap Y=\emptyset$. For large enough $k$, we let $h=h_{2}^{k} f^{2} h_{2}^{-k}$. Then the subgroup $\left\langle h, f_{i}\right\rangle$ is nonelementary for each large $i$.

In either case, there is a $g$-elliptic element $h$ such that $\left\langle h, f_{i}\right\rangle$ is nonelementary for each large $i$. Thus, $\left\langle h, f_{i}\right\rangle$ is discrete, which violates Lemma 2.6 since $f_{i} \rightarrow I$ as $i \rightarrow \infty$. The proof is complete.

Proof of Theorem 3.2. Suppose that $G$ is not discrete. Then by Proposition 5.2, $G$ contains a sequence $\left\{f_{i}\right\}$ such that each $f_{i}$ is $g$-elliptic and

$$
f_{i} \rightarrow I \quad \text { as } i \rightarrow \infty
$$

Since $W Y(G)=W(G)$ is finite, we know that there is a parabolic element $g$ with $\operatorname{Fix}(g) \cap \operatorname{Fix}\left(f_{i}\right)=\emptyset$ for all large $i$ by choosing a suitable subsequence if needed. Then for each large $i$, there is an integer $m_{i}$ such that $f_{i} g^{m_{i}}$ is loxodromic. Since $\left\langle g, f_{i}\right\rangle=\left\langle g, f_{i} g^{m_{i}}\right\rangle$ is nonelementary, it is discrete by our assumption. This violates Lemma 2.6.

Proof of TheOREM 3.3. We assume that $\sigma(L(G))=\overline{\mathbb{R}}^{k}$, where $1 \leq k \leq n$. Suppose that $G$ is not discrete. Then Lemma 2.13 implies that $\Phi^{+}(G)=\{g \in \Phi(G) \mid g$ is sense preserving $\}$ is dense in $\operatorname{PSL}\left(2, \Gamma_{k}\right)$.

Since $G$ contains a $g$-elliptic element $f$ which is not an element of $W Y(G)=$ $W(G)$, there is a parabolic element $p$ in $G$ with $\operatorname{Fix}(p) \cap \operatorname{Fix}(f)=\emptyset$. By the hypotheses, we see that $\langle p, f\rangle$ is discrete. Conjugate $G$ so that $p(\infty)=\infty$. It follows 
that $f(\infty) \neq \infty$. By [17] and the proof of Theorem 2.9, we may assume that $p(x)=A x+a$, where $A a=a, a \in \mathbb{R}^{k} \times\{0\}$,

$$
A=\left(\begin{array}{cc}
A_{k} & 0 \\
0 & A_{n-k}
\end{array}\right),
$$

$A_{k} \in O(k)$ and $A_{n-k} \in O(n-k)$.

If $A$ is a rational rotation, then we can choose $p$ to be a translation and conjugate it such that $p(x)=x+1$. In this case, we let $p_{i}(x)=x+1$.

If $A$ is an irrational rotation, then we may assume that $a=1$ and $A^{k_{i}} \rightarrow E_{n}$ as $i \rightarrow \infty$. Let $h_{i}(x)=x / k_{i}$. Then $h_{i} p^{k_{i}} h_{i}^{-1}(x)=A^{k_{i}} x+1 \rightarrow x+1$ as $i \rightarrow \infty$. Since the restriction of each $h_{i}$ to $\overline{\mathbb{R}}^{k}$ is an element of $\operatorname{PSL}\left(2, \Gamma_{k}\right)$, there is a sequence $\left\{h_{i_{j}}\right\} \subset \Phi^{+}(G)$ such that $\left.h_{i_{j}} \rightarrow h_{i}\right|_{\overline{\mathbb{R}}^{k}}$ as $j \rightarrow \infty$. Then $\widetilde{h_{i_{j}}} \rightarrow h_{i}$ as $j \rightarrow \infty$, where $\widetilde{h_{i j}} \subset \operatorname{PSL}\left(2, \Gamma_{n}\right)$ is the Poincaré extension of $h_{i_{j}}$ to $\overline{\mathbb{R}}^{n}$. For each $i$, we choose an element from $\left\{h_{i_{j}}\right\}$, which is denoted by $h_{i_{i}}$. Then $\widetilde{h_{i_{i}}} p^{k_{i}} \widetilde{h_{i_{i}}^{-1}}(x) \rightarrow x+1$ for any $x \in \overline{\mathbb{R}}^{n}$. For each $h_{i_{i}}$, we choose an element $f_{i} \in \Phi^{-1}\left(h_{i_{i}}\right) \subset G$. Then by Theorem 2.9, $f_{i}=f_{i_{i}} \widetilde{h_{i_{i}}}$, where $\left.f_{i_{i}}\right|_{\overline{\mathbb{R}}^{k}}=I$. By choosing a subsequence if necessary, we may assume that $f_{i_{i}}(x) \rightarrow D x$, where

$$
D=\left(\begin{array}{cc}
E_{k} & 0 \\
0 & D_{n-k}
\end{array}\right)
$$

and $D_{n-k} \in O(n-k)$. Now we consider the sequence $\left\{f_{i} p^{k_{i}} f_{i}^{-1}\right\}$. Since

$$
f_{i} p^{k_{i}} f_{i}^{-1}=f_{i_{i}} \widetilde{h_{i_{i}}} p^{k_{i}} \widetilde{h_{i_{i}}^{-1}} f_{i_{i}}^{-1},
$$

we have that $f_{i} p^{k_{i}} f_{i}^{-1}(x) \rightarrow x+1$. In this case, we let

$$
p_{i}(x)=f_{i} p^{k_{i}} f_{i}^{-1}(x) .
$$

Since $\infty \notin \operatorname{Fix}(f)$, we know by [4] and the proof of Theorem 2.9 that $f$ has the following form

$$
f(x)=t^{2} B \frac{x-b}{|x-b|^{2}}+c,
$$

where $b, c \in \mathbb{R}^{k} \times\{0\}, t>0$,

$$
B=\left(\begin{array}{cc}
B_{k} & 0 \\
0 & B_{n-k}
\end{array}\right),
$$

$B_{k} \in O(k)$ and $B_{n-k} \in O(n-k)$.

Choose $g(x)=N^{2} x$ so that $N^{2} t>1$. Then

$$
g f g^{-1}(x)=t^{2} N^{4} B \frac{x-N^{2} b}{\left|x-N^{2} b\right|^{2}}+N^{2} c .
$$


Since the restriction of $g$ to $\overline{\mathbb{R}}^{k}$ is an element of $\operatorname{PSL}\left(2, \Gamma_{k}\right)$, there is a sequence $\left\{g_{i}\right\} \subset \Phi^{+}(G)$ such that $\left.g_{i} \rightarrow g\right|_{\overline{\mathbb{R}}^{k}}$. Thus, $\tilde{g}_{i} \rightarrow g$. For each $g_{i}$, we choose an element $h_{i} \in \Phi^{-1}\left(g_{i}\right) \subset G$. Then by Theorem 2.9, $h_{i}=h_{i_{0}} \tilde{g}_{i}$, where $\left.h_{i_{0}}\right|_{\overline{\mathbb{R}}^{k}}=I$. By choosing a subsequence if necessary, we may assume that $h_{i_{0}}(x) \rightarrow T x$,

$$
T=\left(\begin{array}{cc}
E_{k} & 0 \\
0 & T_{n-k}
\end{array}\right),
$$

where $T_{n-k} \in O(n-k)$. This implies that

$$
h_{i} f h_{i}^{-1}=h_{i_{0}} \widetilde{g}_{i} f \widetilde{g_{i}^{-1}} h_{i_{0}}^{-1}(x) \rightarrow t^{2} N^{4} T B T^{-1} \frac{x-N^{2} b}{\left|x-N^{2} b\right|^{2}}+N^{2} c \quad \text { as } i \rightarrow \infty .
$$

We consider the sequence $\left\{H_{i}=\left\langle p_{i}, h_{i} f h_{i}^{-1}\right\rangle\right\}$. Each $H_{i}$ is a nonelementary subgroup of $G$ generated by a parabolic and a $g$-elliptic element and hence discrete by assumption. Let $p_{0}(x)=x+1$ and

$$
f_{0}(x)=t^{2} N^{4} T B T^{-1} \frac{x-N^{2} b}{\left|x-N^{2} b\right|^{2}}+N^{2} c .
$$

Then $H=\left\langle p_{0}, f_{0}\right\rangle$ is the algebraic limit of $\left\{H_{i}\right\}$. By Proposition 5.3, $\left\{H_{i}\right\}$ satisfies the E-condition. Hence, Lemma 6.1 yields that $H$ is a Kleinian group. By Lemma 6.2, the radii of the isometric spheres of $g \in H-\operatorname{Stab}_{H}(\infty)$ are not larger than one, which is a contradiction with the assumption $N^{2} t>1$. The proof is complete.

\section{References}

[1] W. Abikoff and A. Haas, 'Nondiscrete groups of hyperbolic motions', Bull. London Math. Soc. 22 (1990), 233-238.

[2] L. V. Ahlfors, 'On the fixed points of Möbius transformations in $\overline{\mathbb{R}}^{n}$, Ann. Acad. Sci. Fenn. Ser. A I Math. 10 (1985), 15-27.

[3] L. Baribeau and T. Ransford, 'On the set of discrete two-generator groups', Math. Proc. Cambridge Philos. Soc. 128 (2000), 245-255.

[4] A. F. Beardon, The Geometry of Discrete Groups, Graduate Text in Mathematics, 91 (Springer, Berlin, 1983).

[5] — 'Some remarks on nondiscrete Möbius groups', Ann. Acad. Sci. Fenn. Ser. A I Math. 21 (1996), 69-79.

[6] M. Chen, 'Discreteness and convergence of Möbius groups', Geom. Dedicata 104 (2004), 61-69.

[7] S. S. Chen and L. Greenberg, Hyperbolic Spaces, Contributions to Analysis (Academic Press, New York, 1974), pp. 49-87.

[8] Y. Chu and X. Wang, 'The discrete and nondiscrete subgroups of $S L(2, \mathbb{R})$ and $S L(2, \mathbb{C})$ ', Hokkaido Math. J. 30 (2001), 649-659.

[9] A. Fang and B. Nai, 'On the discreteness and convergence in $n$-dimensional Möbius groups', J. London Math. Soc. 61 (2000), 761-773.

[10] J. Gilman, 'Inequalities in discrete subgroups of $\operatorname{PSL}(2, \mathbb{R})$ ', Canad. J. Math. 40 (1988), 114-130.

[11] S. Hersonsky, 'A generalization of the Shimizu-Leutbecher and Jørgensen inequalities to Möbius transformations in $\mathbb{R}^{N}$, Proc. Amer. Math. Soc. 121 (1994), 209-215. 
[12] N. A. Isachenko, 'Systems of generators of subgroups of PSL(2, $\mathbb{R})$ ', Siberian Math. J. 31 (1990), $162-165$.

[13] T. Jørgensen, 'On discrete groups of Möbius transformations', Amer. J. Math. 98 (1976), 739-749.

[14] _ 'A note on subgroups of SL(2, C)', Quart. J. Math. Oxford 28 (1977), 209-212.

[15] G.J. Martin, 'On discrete Möbius groups in all dimensions', Acta Math. 163 (1989), 253-289.

[16] D. Sullivan, 'Quasiconformal homeomorphisms and dynamics II: structural stability implies hyperbolicity for Kleinian groups', Acta Math. 155 (1985), 243-260.

[17] P. Tukia, 'Differentiability and rigidity of Möbius groups', Invent. Math. 82 (1985), 557-578.

[18] P. Tukia and X. Wang, 'Discreteness of subgroups of SL(2, $\mathbb{C})$ containing elliptic elements', Math. Scand. 91 (2002), 214-220.

[19] X. Wang, 'Dense subgroups of $n$-dimensional Möbius groups', Math. Z. 243 (2003), 643-651.

[20] , 'Algebraic convergence theorems of $n$-dimensional Kleinian groups', Israel J. Math. 162 (2007), 221-233.

[21] X. Wang, L. Li and W. Cao, 'Discreteness criteria for Möbius groups acting on $\overline{\mathbb{R}}^{n}$, Israel J. Math. 150 (2005), 357-368.

[22] X. Wang and W. Yang, 'Discreteness criteria of Möbius groups of high dimensions and convergence theorem of Kleinian groups', Adv. Math. 159 (2001), 68-82.

[23] - 'Discreteness criteria for subgroups in $\mathrm{SL}(2, \mathbb{C})$ ', Math. Proc. Cambridge Philos. Soc. 124 (1998), 51-55.

[24] - 'Dense subgroups and discrete subgroups in SL(2, C)', Quart. J. Math. Oxford 50 (1999), $517-521$.

[25] _ 'Generating systems of subgroups in PSL(2, $\left.\Gamma_{n}\right)$ ', Proc. Edinburgh Math. Soc. 45 (2002), $49-58$.

[26] S. C. Wang and Q. Zhou, 'On the proper conjugation of Kleinian groups', Geom. Dedicata 50 (1995), 1-10.

[27] P. L. Waterman, 'Möbius transformations in several dimensions', Adv. Math. 101 (1993), 87-113.

[28] _ 'Purely elliptic Möbius groups', in: Holomorphic Functions and Moduli, Vol. II (Berkeley, CA, 1986), Mathematical Sciences Research Institute Publications, 11 (Springer, New York, 1988), pp. 173-178.

[29] N. J. Wielenberg, 'Discrete Möbius groups: fundamental polyhedra and convergence', Amer. J. Math. 99 (1977), 861-877.

[30] S. Yang, 'On the discreteness in SL(2, C)', Math. Z. 255 (2007), 227-230.

LIU-LAN LI, Department of Mathematics, Hengyang Normal University, Hengyang, Hunan 421008, P.R. China

e-mail: lanlimail2008@yahoo.com.cn

XIAN-TAO WANG, Department of Mathematics, Hunan Normal University Changsha, Hunan 410081, P.R. China

e-mail: xtwang@hunnu.edu.cn 